\title{
Projeto Amora: a possibilidade de aprender matemática utilizando redes sociais apoiados nas teorias de Raymond Duval e Vygotsky
}

Rodrigo Sychocki da Silva*

Resumo: Neste artigo relatamos a experiência de trabalho feita com um grupo de alunos do colégio de Aplicação da UFRGS ocorrido no segundo semestre de 2006. As atividades foram ministradas na oficina de Interação Virtual e a presença das redes sociais naquela época foram determinantes para a ocorrência das atividades. O uso da internet como ferramenta de busca foi importante ao propor a sequência de atividades, pois possibilitou que os alunos desenvolvessem autonomia pela sua aprendizagem. A fundamentação teórica é feita com base nos estudos de Raymond Duval, que considera a questão dos registros de representação semiótica como hipótese para investigar os processos de aprendizagem. Ele apresenta a especificidade da aprendizagem e do ensino da matemática ligadas aos aspectos semióticos das representações matemáticas. Através dos estudos de Duval apresenta-se a possibilidade de inserir redes virtuais no ensino de alguns conteúdos de matemática propostos no currículo. A teoria da zona do desenvolvimento proximal de Vygotsky foi usada para reconhecer a importância da função do colega de aula durante o decorrer das atividades em aula, proporcionando uma aprendizagem mútua dos alunos.

Palavras-chave: Aprendizagem; Representação Semiótica; Redes Virtuais; Tecnologia.

* Mestre em Ensino de Matemática. Doutorando no PGIE da UFRGS. Professor do Instituto Federal do Rio Grande do Sul - Campus Caxias do Sul. E-mail: rodrigo. silva@caxias.ifrs.edu.br 


\begin{abstract}
In this paper we report the experience of work done with a group of students of the school's Application UFRGS during the second half of 2006. The activities were held in the workshop of virtual interaction and the presence of social networks at the time were instrumental in the occurrence of activities. The use of the Internet as a search tool was important to propose a sequence of activities, because it enabled students to develop autonomy in their learning. The theoretical framework is based on studies, of Raymond Duval considers the question of semiotic registers of representation, as a hypothesis to investigate the learning processes. He presents the specificity of learning and teaching of mathematics related to semiotic aspects of mathematical representations. Through studies of Duval presents the possibility of inserting virtual networks in teaching some contents of the proposed math's curriculum. The theory of zone of proximal development Vygotsky was used to recognize the important function of the classmate during the course of class activities, providing a mutual learning of students.
\end{abstract}

Keywords: Learning; Semiotic Representation; Virtual Networks; Technology.

\title{
Introdução
}

Este trabalho relata o processo de aplicação de duas atividades realizadas na oficina de Interação Virtual no colégio de Aplicação da Universidade Federal do Rio Grande do Sul no segundo semestre de 2006. O público alvo dessa proposta foi alunos do ensino fundamental, no nível da $5^{\mathrm{a}}$ e $6^{\mathrm{a}}$ séries. A motivação para a escrita deste texto foi mostrar que, na época da realização dessas atividades, foi feito o uso de redes virtuais para contribuir na aprendizagem dos alunos, mesmo que na época não houvesse a divulgação das ideias sobre aprendizagem utilizando esse tipo de mecanismo que há atualmente.

A proposta da Interação Virtual é integrar ao currículo assuntos de matemática que são possíveis de trabalhar com o uso dos recursos computacionais. Ao propor as sequências de 
atividades, a preocupação dos docentes envolvidos no projeto é a de proporcionar aos alunos a maior compreensão dos assuntos de matemática abordados durante a oficina. Durante todo um semestre, com a orientação do Instituto de Matemática da UFRGS e promovida por acadêmicos do curso de Licenciatura em Matemática, são desenvolvidas atividades no turno inverso ao das aulas tradicionais, visando a um aprofundamento dos tópicos abordados nas aulas.

Com a preocupação de tornar possível a aprendizagem efetiva da matemática, na oficina de Interação Virtual, desenvolvem-se fortemente as ideias das representações semióticas dos objetos matemáticos. Para fundamentar essas ideias, utilizamos a teoria de Duval, sobre as representações semióticas que os alunos fazem para compreender os conceitos abordados em uma aula. Ao propor as atividades, nota-se que a colaboração entre os alunos ocorre mutuamente, uma vez que eles são organizados em duplas (ou trios) para desenvolver as sequências de atividades. Percebe-se, nesse momento, a forte influência da zona de desenvolvimento proximal, inicialmente proposto por Vygotsky, em que a aprendizagem é construída através de relações estabelecidas em uma vizinhança próxima. O professor torna-se um elemento importante que serve como mediador dessas relações, proporcionando que ocorra efetivamente o momento de aprendizagem dos alunos.

Uma das atividades é sobre a teoria dos conjuntos, em que apresentamos uma situação prática para o uso dessa teoria. A segunda atividade é sobre um levantamento de informações na Internet, com a finalidade de fazer a construção de gráficos e interpretação dos dados utilizando o software Excel. Ambas as atividades despertaram interesse nos alunos, pois estavam sendo abordadas partindo de situações de modelagem matemática que faziam sentido para eles. 


\section{Alguns aspectos teóricos envolvidos}

Duval (1996) afirma que o processo de aprendizagem na matemática não pode ser considerado como o mesmo de aprender outras disciplinas. A matemática pressupõe como requisito uma atividade cognitiva diferente de outras áreas de conhecimento. Duval apresenta a importância e a diferenciação das representações semióticas utilizadas na matemática, uma vez que as representações exercem diferentes funções, tais como comunicação, desenvolvimento das representações mentais e produção de conbecimento.

Os argumentos propostos por Duval afirmam que para representar, tratar e converter em representações semióticas é necessário mobilizar sistemas cognitivos específicos para cada uma das atividades propostas aos alunos. Para o autor, somente pode-se aprender efetivamente matemática através das representações semióticas dos objetos matemáticos. Durante o processo de aprendizagem é importante que o aluno saiba manipular as representações semióticas, com a finalidade de transformá-las em outras, se necessário. Isso fornece subsídios para uma aprendizagem efetiva de um dado problema.

Duval (2003) argumenta que não se deve confundir um objeto e sua representação, uma vez que, realizando operações em mais de um sistema de representação, é implícito o entendimento de que nenhuma das representaçôes consideradas é propriamente o objeto matemático, mas apenas estamos diante de um representante, um ente que está "no lugar dele" com a finalidade de permitir o acesso aos objetos matemáticos. Por exemplo, o desenho de uma circunferência, a palavra circunferência, a equação de uma circunferência, são todas representações diferentes que se referem ao mesmo objeto "circunferência", mas nenhuma das representações de fato é a circunferência, apenas estão representando. São considerados relevantes os registros que permitem o acesso ao objeto e ao tratamento do objeto matemático.

Ainda em Duval (2003, p. 14) nota-se que "a originalidade da atividade matemática está na mobilização simultânea 
de ao menos dois registros de representação ao mesmo tempo, ou na possibilidade de trocar a todo o momento de registro de representação". Portanto, as atividades cognitivas envolvidas no ensino e na aprendizagem da matemática requerem regras de codificação próprias. Cada um dos registros possui limitações representativas específicas, surgindo, portanto, a necessidade da utilização de outros sistemas de expressão e de representação, além da linguagem usual e de imagens, como ferramenta para a real compreensão do conceito matemático. Considerando a possibilidade de representar os objetos conceituais matemáticos, Duval afirma que a noção de tratamento e de conversão são operaçôes cognitivas diretamente envolvidas durante o processo de compreensão do conhecimento matemático, isto é, na construção dos conceitos.

Para a aprendizagem de matemática é importante salientar que a movimentação nos registros é a chave para a solução dos problemas cognitivos encontrados pelo professor durante a aula. Ao escolher o registro mais adequado para aplicar os tratamentos, implica primeiramente o desenvolvimento do raciocínio, consequentemente, leva à resolução correta dos problemas matemáticos e, finalmente, conduz à aprendizagem. A necessidade da diferenciação entre o objeto matemático e sua representação constitui-se em um aspecto de relevância para Duval (1993, p. 51), pois "a compreensão de um conteúdo conceitual repousa sobre a coordenação de ao menos dois registros de representação, e esta coordenação se manifesta pela rapidez e a espontaneidade da atividade cognitiva de conversão.”. Dispondo de vários registros é possível estabelecer a distinção essencial do objeto matemático em relação ao registro na representação semiótica, ou seja, estabelecer a distinção entre o representante e o representado.

Pode-se dizer que ocorre o processo de conceitualização quando o aluno é capaz de identificar um registro de representação semiótica do objeto matemático estudado, escolhido em um conjunto onde há vários registros apresentados, de maneira a favorecer a resolução de um dado problema. Para entender a 
proposta de Duval, inicialmente deve-se perceber o quanto a matemática é dependente das representações que são utilizadas para ter acesso a ela e, em segundo lugar, o professor deve saber proporcionar estratégias específicas para o trabalho pedagógico com essa disciplina.

Ainda é importante ressaltar que as atividades norteadoras do Projeto Amora objetivam uma reestruturação curricular, destacando o papel desempenhado pelo professor e sua importância na aprendizagem dos alunos através da construção compartilhada de conhecimentos com o uso de projetos de aprendizagem e proporcionando a inserção das tecnologias de informação e comunicação ao currículo escolar. Ao propor o uso das tecnologias, tem-se que as trocas de informações entre os alunos proporcionam uma aprendizagem efetiva durante as aulas.

A zona de desenvolvimento proximal (ZDP) apresentada por Vygotsky surge na proposta pedagógica do Projeto Amora, pois é necessário que o professor coloque em prática tarefas de ensino que potencializem a aprendizagem dos alunos. Os instrumentos que o professor pode usar são a linguagem e o contexto cultural, os quais Vygotsky considera como ferramentas essenciais que estão a serviço da aprendizagem e do desenvolvimento. Além disso, o professor é o mediador entre a criança e os objetos matemáticos, a fim de proporcionar uma aprendizagem de qualidade.

Ao propor algumas tarefas que estão além da zona de desenvolvimento proximal é muito provável que a criança não irá compreender corretamente, ela não vai ser capaz de realizá-las ou realiza incorretamente. Nesse momento, os colegas de aula se tornam mediadores que se revelam eficazes na aprendizagem dos demais colegas de aula. Pensando nisso, quando o professor propõe a criação de grupos de aprendizagem colaborativa, com crianças situadas em diferentes níveis de aprendizagem, embora estejam próximas na capacidade para a realização das tarefas, isso constitui uma estratégia de mediação importante na aprendizagem. Importa que o papel do mediador externo deve ter significado para a criança, deve estar conectado a um objeto 
que a criança use durante o desempenho da tarefa e combine a mediação com o uso da linguagem e do contexto cultural.

Embasado na representação semiótica proposta por Duval e na presença da zona de desenvolvimento proximal proposta por Vygotsky, o grupo de acadêmicos na Interação Virtual em 2006 tinha a preocupação de propor aos alunos do Projeto Amora atividades que relacionassem matemática com cotidiano e utilizassem a tecnologia durante a sua execução. Isso foi e continua sendo importante para a inserção de novos conteúdos na estrutura do currículo e também mostra a possibilidade de o aluno conceber que a matemática possui inúmeras aplicações além dos tradicionais cálculos desenvolvidos em aula, os quais também são fundamentais para a sua formação durante a vida escolar.

\section{O encaminhamento da proposta}

Os professores da oficina da interação virtual propõem aos alunos que participam uma ampla visão nos conteúdos de matemática, e a utilização dos recursos tecnológicos da informática serve para estabelecer a conexão entre a matemática e o cotidiano. Ao longo das aulas são propostas inúmeras atividades, que ficam dispostas em um ambiente de livre acesso na Internet. Ao acessar a página inicial, conforme mostra a Figura 1, o aluno é levado para uma página da Web onde ele encontrará os subsídios necessários para o desenvolvimento das tarefas propostas. 
Figura 1: Página inicial da Interação Virtual em 2006/1.

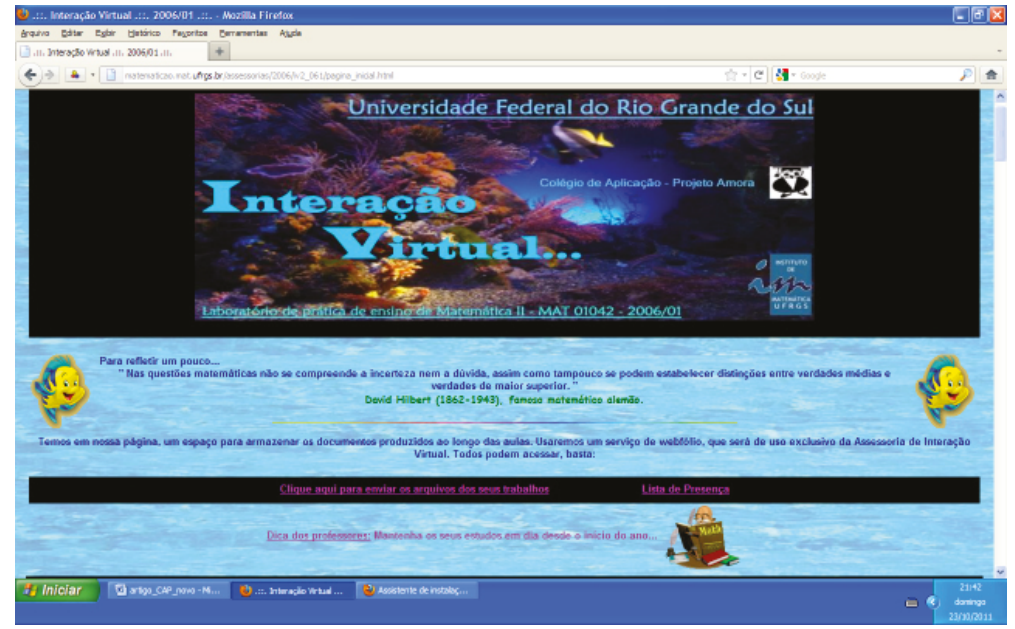

Na época do desenvolvimento das atividades dessa oficina, uma rede social muito comum e divulgada entre os alunos era o ambiente do Orkut. Ele foi usado para trabalhar ideias muito básicas sobre os conjuntos matemáticos, com o foco principal nas operações elementares. Nessa atividade, inicialmente foram abordadas as seguintes operações: união, intersecção e diferença de conjuntos.

Para o desenvolvimento da atividade sobre conjuntos foi utilizada a ideia de criar um contexto que envolvesse os alunos em uma situação: o uso do ambiente Orkut para aprender conceitos de matemática. A noção de "comunidade virtual" foi usada para mostrar aos alunos que no Orkut era possível aprender matemática. Após os alunos explorarem algumas comunidades específicas no Orkut, que o grupo de professores recomendou, eles foram conduzidos até as atividades, como mostra a Figura 2. 
Figura 2: Página com as atividades sobre conjuntos no Orkut.

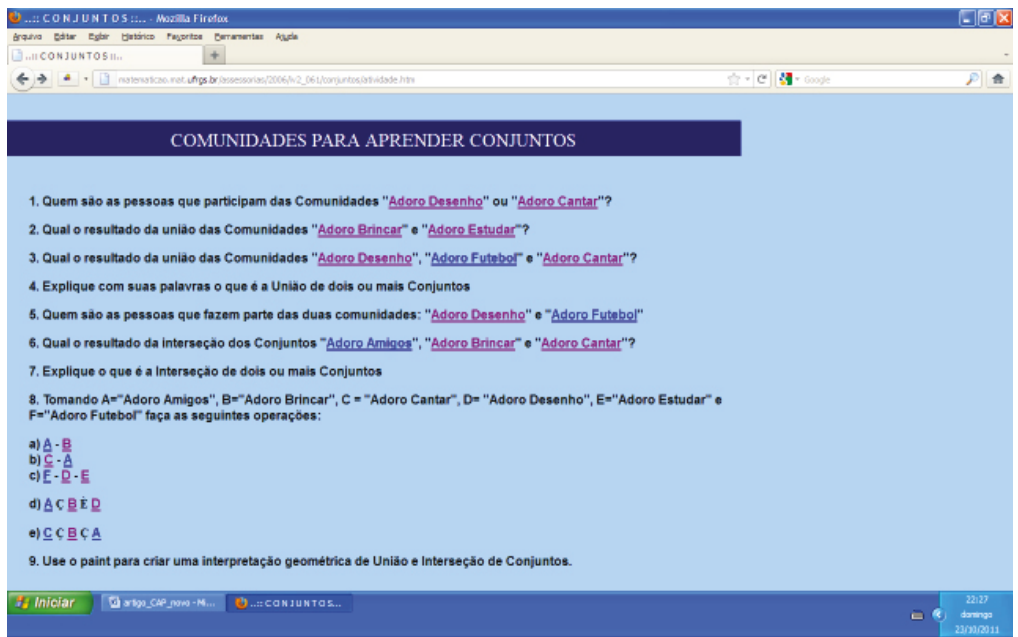

Em um segundo momento, o grupo docente utilizou as chaves dos grupos da Copa do Mundo 2006 e também o Microsoft Excel para explorar as propriedades de uma ferramenta chamada filtro. Alguns aspectos sobre a Copa do Mundo de 1998 foram utilizados para ensinar aos alunos noções um pouco mais avançadas sobre conjuntos como inclusão de conjuntos e ideias de completudel, conforme Figura 3.

1 Completude é a ideia de que toda sentença definida em um conjunto pode ser provada. Ou equivalentemente, que a sua negação pode ser provada a partir de certa teoria. 
Figura 3: Página com as atividades sobre conjuntos na Copa do Mundo.

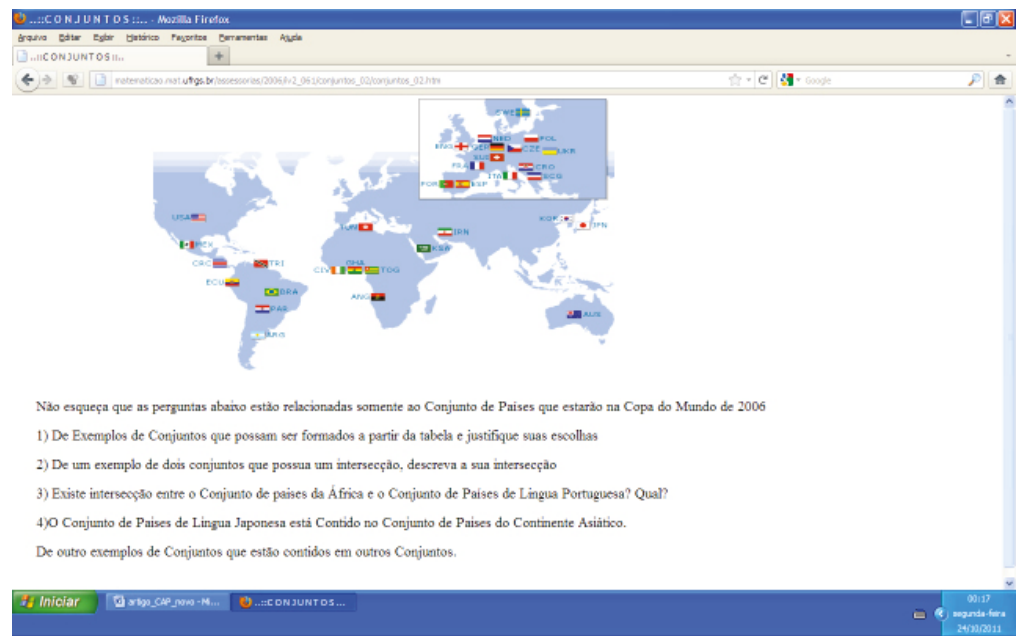

De um modo geral, a atividade sobre conjuntos, produziu resultados diferentes entre os alunos do Projeto Amora. Considera-se que a ideia de usar o ambiente virtual Orkut foi satisfatória, pois os alunos apresentaram facilidade em assimilar os conceitos básicos trabalhados. Ressalta-se que os alunos apresentaram dificuldades quando se iniciou o trabalho com as noções mais formais da teoria, como por exemplo, a inclusão de conjuntos. Essa noção foi introduzida utilizando a ferramenta de "filtro" no Microsoft Excel e foram reforçadas por meio de questões que despertaram o raciocínio dos alunos.

Em seguida, pensando em trabalhar noções sobre gráficos, onde a coleta e interpretação dos dados obtidos foram as primeiras ideias desenvolvidas no trabalho, utilizaram-se alguns recursos disponíveis no Microsoft Excel, conforme Figura 4. O objetivo principal dessa atividade foi inserir o aluno em uma situação cotidiana (pesquisa de preço) no ambiente virtual. Foram utilizados alguns sites que serviram como guia para pesquisa dos preços de alguns produtos previamente listados. 
Figura 4: Atividade inicial sobre gráficos e pesquisa na Internet.

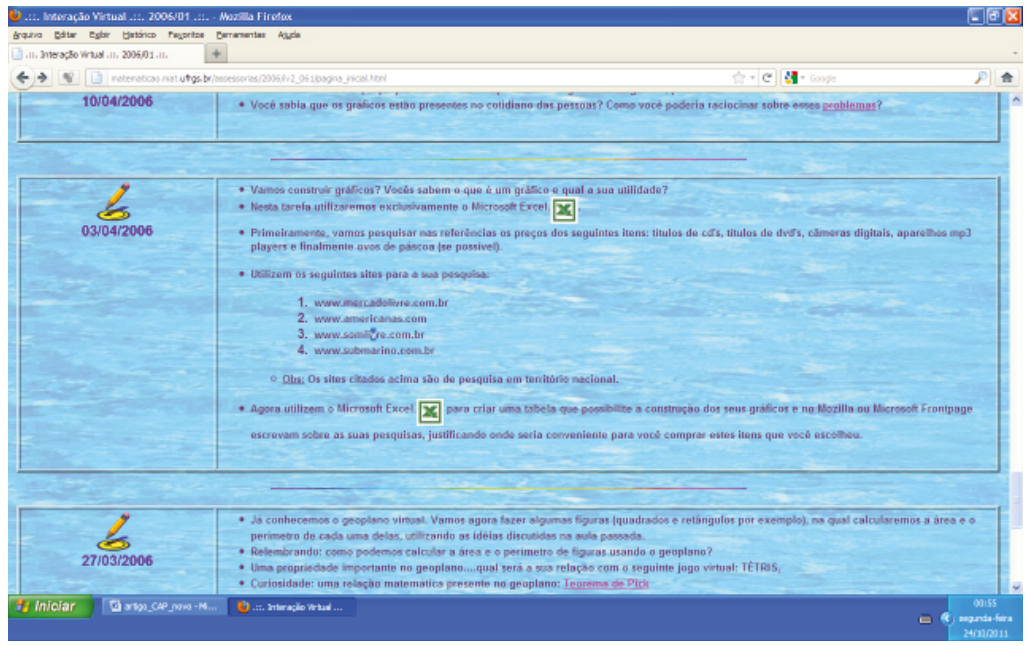

De um modo geral os resultados obtidos com essa atividade foram bons. Alguns poucos alunos possuíram dificuldades para compreender o conceito matemático sobre os gráficos, uma vez que era o primeiro contato deles com esse assunto. Contudo, a maioria dos alunos não possuiu dificuldades, nem no momento da pesquisa quanto no momento da análise dos gráficos construídos no Microsoft Excel. Podemos concluir que a atividade foi considerada muito interessante por parte dos alunos, pois foi muito qualitativo adaptar uma situação prática com o uso de um recurso virtual para interpretá-lo. Sugere-se fortemente que essa mesma atividade possa ser retomada em outras séries da escola básica, daí envolvendo tipos específicos de gráficos e situações.

Após essa atividade inicial, os alunos foram conduzidos para a resolução de problemas menos elementares sobre análise e interpretação de gráficos, conforme Figura 5. Foram explorados basicamente três tipos de gráficos: setor circular, histograma e poligonal. Cada problema proposto procurava apresentar uma situação cotidiana para o uso desse conteúdo matemático na resolução e interpretação das informações. 
Figura 5: Problemas diversos sobre gráficos.

\section{Questões para análise...}

01) Em Porto Alegre foi realizada uma pesquisa com 1000 pessoas. O resultado da pesquisa pode ser percebido no gráfico abaixo:

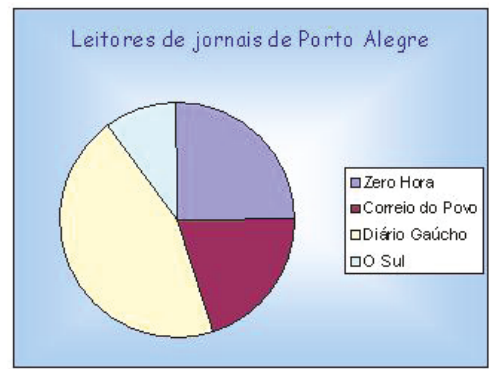

Somente sabemos que o número de pessoas que lê o jornal Diário Gaúcho é 450. O que você pode dizer sobre os demais leitores? É possível calcular o número de leitores dos outros jornais? Justifique.

02) Em três lojas de roupa de um Shopping de Porto Alegre foi realizada uma consulta de preços. O gráfico abaixo representa o

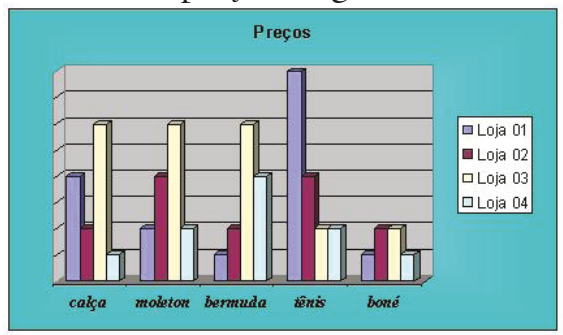

Sabendo que o par de tênis na loja 01 custa 400 reais, o que podemos dizer sobre os outros preços? Descreva o seu raciocínio e verifique a quantidade mínima de dinheiro que uma pessoa precisa ter para comprar todos os itens. 
03) O IBAMA, órgão federal que controla o número de animais e plantas no Brasil, fez uma pesquisa sobre alguns animais de estimação que são "diferentes" nas casas dos brasileiros durante o passar dos anos. O resultado pode ser visualizado no gráfico abaixo:

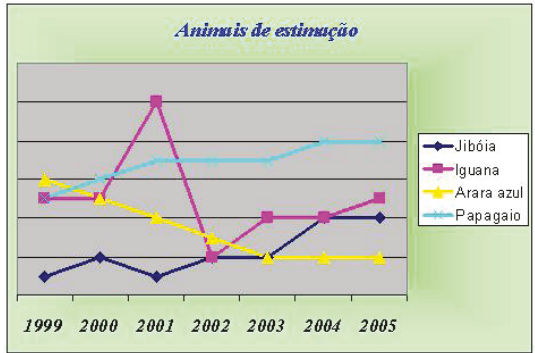

Sabendo somente que o número de Iguanas atingiu o seu valor máximo no ano de 2001 que foi exatamente 100 . O que você pode afirmar sobre como foi a variação ocorrida com os outros animais?

Nessa atividade os resultados foram satisfatórios e os alunos a realizaram muito bem, sem maiores problemas. $\mathrm{O}$ grupo não apresentou dificuldades em entender a proposta de cada uma das questões. Essa atividade foi muito qualitativa, pois possibilitou o desenvolvimento de criação e interpretação de dados organizados em um gráfico. Certamente essa ideia é ideal para ser praticada com outros alunos eventualmente, em atividades que envolvem o uso do computador para aprender matemática.

No desenvolver dessas duas propostas, os alunos apresentaram autonomia e comprometimento com a aprendizagem. Através das representações semióticas, foram capazes de formular hipóteses, conjecturar e verificar a sua validade através de raciocínios matemáticos. Ao escolher a melhor representação do objeto matemático que estava sendo trabalhado foi possível identificar que efetivamente estava ocorrendo aprendizagem daquele conteúdo. Com a possibilidade de interação entre os alunos, verificou-se que a influência do raciocínio presente na dupla ou no trio de alunos interfere na aprendizagem individual de cada 
um, isto é, segundo Vygotsky é possível aprender interagindo com os vizinhos, através de perguntas, deduções e raciocínios. Com isso podem os alunos chegar a conclusões matemáticas corretas após percorrer algumas etapas no desenvolvimento e amadurecimento das ideias.

\section{Considerações finais}

O Projeto Amora possibilita que os docentes desenvolvam maneiras de abordar a matemática na forma mais flexível e dinâmica. Em particular, foi possível inserir as redes sociais para o ensino de conteúdos considerados tradicionais por parte de alguns docentes da escola básica. Os resultados dessa inserção foram satisfatórios, uma vez que produziu a aprendizagem dos conceitos apresentados na ocasião.

Ao possibilitar que o aluno faça representações, analise as situações apresentadas, formule hipóteses, teste-as utilizando critérios pré-determinados e chegue às próprias conclusões, o professor está cumprindo o seu papel de orientador no processo de aprendizagem.

Desde que o Projeto Amora foi iniciado, um grande número atividades vem sendo desenvolvido e aplicado no Colégio de Aplicação, pensando-se no desenvolvimento das habilidades cognitivas dos alunos. Isso possibilita tornar o currículo da escola rico e com grandes contribuições para a vida pós-escola, fazendo com que ele se torne peça principal na formação dos alunos.

\section{Referências}

DUVAL, Raymond. Registre de représentation sémiotique et fonctionnement cognitif de la pensée. Annales de Didactique et Sciences Cognitives. Strasbourg: IREM - ULP, v. 5, p. 37-65, 1993. 
. Quel cognitif retenir en didactique des mathématiques? Recherches en Didactique Mathématiques (RDM), v. 16, n. 3, p. 349-382, 1996.

. Registros de representação semiótica e funcionamento cognitivo da compreensão em matemática. In: MACHADO, Silvia Dias Alcântara (Org.). Aprendizagem em matemática: registros de representação semiótica. Campinas: Papirus, 2003, p. 11-33.

INTERAÇÃO VIRTUAL 2006/1. Projeto Amora. Disponível em: <http:// matematicao.mat.ufrgs.br/assessorias/2006/iv2_061/pagina_inicial. html>. Acesso em: 10 out. 2011.

VYGOTSKY, Lev Semenovitch. Teoria e método em psicologia. São Paulo: Martins Fontes, 1996.

. A consciência como problema da psicologia do comportamento. In: Teoria e método em psicologia. São Paulo: Martins Fontes, 1996. . A formação social da mente. São Paulo: Martins Fontes, 1998. 